\title{
Conocimiento sobre el ácido fólico en la prevención de defectos de cierre del tubo neural: una encuesta a mujeres que viven en Santiago de Chile
}

\author{
Rosa A Pardo $\mathbf{V}^{\mathbf{1}, 2}$, G uillermo Lay-Son $\mathbf{R}^{\mathbf{1}}$, Waldo Aranda \\ $\mathrm{Ch}^{3}$, Martín Dib Ma, Pablo Espina $\mathrm{M}^{\mathrm{a}}$, María J Muñoz $\mathrm{K}^{\mathrm{a}}$, \\ Pilar Muñoz Ba, María F Navarrete Ca, Natalia V O jeda \\ $M^{a}$, Jorge Parra ${ }^{a}$, Cristián A Rebolledo Da, Pedro Recabal \\ $\mathrm{G}^{\mathrm{a}}$, Tamara Tenhamm $\mathrm{T}^{\mathrm{a}}$. \\ Awareness of post partum women
on the effects of folic acid in the
prevention of congenital anomalies
}

Background: Wheat flour in Chile is fortified with folic acid and pregnant women are also supplemented with the vitamin, but the population level of knowledge or awareness about this vitamin and its use by pregnant women is unknown. Aim: To assess the level of knowledge that postpartum women from Santiago de Chile have about folic acid. Material and methods: A questionnaire about folic acid and its efects on the prevention of neural tube defects was developed adapting questionnaires designed abroad. It was applied by medical students to puerperal women, hospitalized in public hospitals. Results: The questionnaire was applied to 342 women aged $26 \pm 7$ years. Sixty one percent were housewives and 55\% completed high school education. Forty seven percent of these women had heard about folic acid, 9.6\% knew that it was able to prevent congenital defects and only one received an adequate supplementation during pregnancy. Women aged 25 to 34 years and those with an adequate medical care during pregnancy had a significantly better knowledge about folic acid and its role in the prevention of congenital anormalies. The more commom means to receive information about folic acid were midwifes (34\%), mass media (28\%) and doctors (20\%). Two bundred eleven women (62\%) agreed to take folic acid in a future gestation and $58 \%$ preferred to do so using fortified foods. Conclusions: Post partum women from Santiago have a poor knowledge about the relevance of folic acid supplementation (Rev Méd Chile 2007; 135; 1551-7).

(Key words: Abnormalities; Folic acid; Nervous system malformations)

\footnotetext{
Recibido el 20 de junio, 2006. Aceptado el 23 de julio, 2007.

${ }^{1}$ Sección de Genética y Unidad de Cuidados Especiales Neonatales, Hospital Clínico Universidad de Chile. ${ }^{2}$ Unidad de Genética, Hospital Dr Sótero del Río. ${ }^{3}$ Escuela de Salud Pública, Universidad de Chile.

${ }^{a}$ Estudiante de Medicina, Universidad de Chile, Santiago de Chile.
}

Correspondencia a: Dra. Rosa A Pardo V. Hospital Clínico Universidad de Chile. Sección de Genética. Santiago de Chile, Chile. Fono-fax: 56-2-9788513. E mail: rpardo@redclinicauchile.cl 
$\mathrm{L}$ os defectos de tubo neural (DTN) son un grupo de anomalías congénitas que aparecen cuando el cierre del tubo neural no ocurre de manera normal, e incluyen la espina bífida, el encefalocele y la anencefalia. Varios estudios han demostrado que la ingesta periconcepcional de acido fólico (AF) reduce la incidencia de $\mathrm{DTN}^{1}$.

En 2000, se inició en Chile el programa de fortificación de la harina de trigo con AF, tras lo cual la tasa de DTN ha disminuido en $40 \%{ }^{2,3}$. Además, en 2003 se promulgó la norma de atención perinatal según la cual es necesario dar suplementación de AF a aquellas mujeres que no reciben la dosis adecuada de prevención a través de su dieta, o bien presentan factores de riesgo para tener hijos con $\mathrm{DTN}^{4}$. Pese a los grandes avances en el área de la fortificación y los incipientes adelantos con la norma de suplementación ${ }^{7}$, en Chile no existen campañas educacionales acerca del uso periconcepcional del AF, ni tampoco hay información referente al nivel de conocimiento que la mujer chilena en edad reproductiva tiene sobre el AF y su asociación con la prevención de anomalías congénitas.

Muchos países han efectuado experiencias de capacitación a mujeres en edad reproductiva y personal de salud sobre AF y gestación, con miras a lograr disminuir la aparición de DTN a través de la suplementación periconcepcional. Las campañas enfocadas a informar a las mujeres han logrado que la suplementación de AF se incremente entre 6 y 50 veces $^{6,7}$ y el resultado tras la intervención en el personal de salud ha aumentado la indicación de la misma en $19 \% 1,8$.

El propósito de este estudio es evaluar, primero, el conocimiento sobre AF de las mujeres en edad reproductiva y segundo, el conocimiento que dichas mujeres tienen de la asociación entre el AF y la prevención de anomalías congénitas en una población de mujeres de Santiago de Chile.

\section{MATERIAL Y MÉTODOS}

Este es un estudio descriptivo, de corte transversal, cuyo protocolo fue aprobado por el Comité de Ética del Hospital Clínico Universidad de Chile.

Sujetos: Los datos fueron recolectados entre agosto y octubre de 2004 en cuatro hospitales públicos de Santiago de Chile (Barros Luco - Trudeau, Luis Tisné, San Borja Arriarán y San Juan de Dios).

Para determinar el tamaño de la muestra se consideró el promedio de partos de cada centro y se empleó un nivel de confianza del 95\% para un error de estimación de 5\% del verdadero valor del parámetro investigado. Luego, la muestra se estratificó en forma proporcional en los 4 centros hospitalarios.

Los criterios de inclusión fueron: consentimiento de participación, encontrarse en los primeros 4 días post-parto, no haber tenido un trabajo de parto prolongado, no haber tenido un niño con malformaciones congénitas, ni encontrarse hospitalizada en la misma habitación con una madre quien hubiese tenido un niño con malformaciones. El trabajo de parto prolongado, fue definido como más de 20 ó 6 horas de trabajo de parto intrahospitalario para mujeres primigestas y multigestas respectivamente. Se consideraron como periodos y dosis adecuados para la suplementación con AF los definidos en la norma ministerial de atención perinatal, a saber: período adecuado de toma de AF, desde 3 meses antes de la concepción hasta las primeras 12 semanas de gestación y dosis adecuada de suplementación con AF, $1 \mathrm{mg}$ diario para las mujeres sin factores de riesgo para tener hijos con DTN y 4 a $5 \mathrm{mg}$ diarios para mujeres con factores de riesgo para tener hijos con DTN (p.e.: mujeres con hijos con DTN, o en tratamiento con antifolínicos, o portadoras de DTN).

Encuesta: Una encuesta referente a conocimientos sobre AF y prevención de anomalías congénitas fue desarrollada basada en la revisión de encuestas validadas en publicaciones internacionales sobre el tema9-11. El cuestionario fue diseñado por 2 médicos locales y probado posteriormente entrevistando 8 mujeres puérperas en el Hospital Clínico Universidad de Chile, tras lo cual se introdujeron modificaciones menores.

La recolección de datos se realizó por estudiantes de medicina, a través de entrevistas cara a cara, utilizando el cuestionario estructurado generado.

El cuestionario tenía varios tipos de preguntas (abiertas, dicotómicas y de alternativa) e incluía datos sociodemográficos; formas de prevención de anomalías congénitas; conocimientos sobre AF 
y su rol en la prevención de anomalías congénitas y uso de suplementación con AF en la última gestación. Además, se recolectaron datos referentes a la ingesta de pan, debido al programa chileno de fortificación de la harina de trigo con $\mathrm{AF}$, materia prima utilizada principalmente en la elaboración de dicho alimento.

Estadística: Se creó una base de datos en el programa EXCEL y el análisis estadístico fue realizado en el programa STATA 7.0. El T-test fue empleado para comparar las variables cuantitativas. El $\mathrm{X}^{2}$ fue utilizado para identificar las asociaciones entre las variables categóricas. La correlación de Pearson fue utilizada para evaluar la relación entre el nivel de conocimiento sobre $\mathrm{AF}$ que tenían las mujeres y variables como: nivel de educación, edad, nacionalidad, paridad y consulta pregestacional. El análisis descriptivo fue usado para describir el conocimiento de las mujeres puérperas sobre el AF. Todas las pruebas fueron realizadas considerando como significativo un valor de $\mathrm{p}<0,05$.

\section{Resultados}

Trescientas cuarenta y dos mujeres puérperas fueron encuestadas entre agosto y octubre de 2004, en las maternidades de cuatro hospitales públicos de Santiago de Chile (Hospital Barros Luco - Trudeau, Hospital Luis Tisné, Hospital San Borja Arriarán y Hospital San Juan de Dios).

La edad media de las mujeres participantes fue de $25,5 \pm 6,8$ años (rango: 15 - 43 años) y sus características sociodemográficas se encuentran en la Tabla 1.

Sólo doce mujeres $(3,5 \%)$ tenían un antecedente médico patológico: una tenía epilepsia, tres tenían diabetes miellitus y ocho diabetes gestacional. Dos casos $(0,6 \%)$ tenían un familiar con DTN (una hermana o hermano con espina bífida). Sin embargo, ninguna mujer con familiares afectados o recibiendo medicamentos anticonvulsivantes recibió una adecuada dosis de suplementación con ácido fólico.

Ochenta y una mujeres $(23,7 \%)$ estaban informadas sobre algún método de prevención de anomalías congénitas, siendo el más frecuentemente referido la no ingesta de alcohol, consumo de tabaco o uso de drogas alucinógenas (Tabla 2).

\section{Tabla 1. Características sociodemográficas de las 342 mujeres participantes en el estudio}

\begin{tabular}{|lcr|}
\hline Característica & Resultados & $\%$ \\
\hline Edad promedio y & & \\
DS (años) & $25,5 \pm 2,68$ & \\
Condición laboral & & \\
Dueña de casa & 210 & 61,4 \\
Estudiante & 43 & 12,6 \\
Otras & 89 & 26,0 \\
Nivel educacional: & & \\
Secundaria incompleta & 92 & 26,9 \\
Secundaria completa & 189 & 55,3 \\
Estudios universitarios & 61 & 17,8 \\
Nacionalidad & & \\
Chilena & 325 & 95,0 \\
Otras & 17 & 5,0 \\
Historia familiar de DTN & 2 & 0,6 \\
Antecedente mórbido: & & \\
Diabetes gestacional & 8 & 2,3 \\
Diabetes & 3 & 0,9 \\
Epilepsia & 1 & 0,3 \\
Pérdidas fetales previas & 68 & 19,9 \\
\hline
\end{tabular}

DS:Desviación estándar. DTN: Defecto de cierre de tubo neural

Ciento sesenta y dos de las participantes (47,5\%) referían haber oído alguna vez sobre el $\mathrm{AF}$, pese a que $16,7 \%$ no recordaba qué información había recibido del mismo, hubo entre las encuestadas quienes sabían que era una vitamina (15\%), que ayudaba a prevenir malformaciones $(9,6 \%)$ y que era necesario ingerirlo durante el embarazo (5,3\%) (Tabla 3).

Las entrevistadas refirieron que sus fuentes de información fueron: las matronas (34\%), los medios de comunicación $(28,4 \%)$, los médicos (20\%), amigos $(9,6 \%)$ y familiares (8\%).

En cuanto a los profesionales de la salud, se detecto que las matronas $(65,5 \%)$ eran quienes más frecuentemente indicaban AF. Pero es de resaltar que del total de la muestra, solamente nueve participantes $(2,6 \%)$ recibieron suplementación adecuada con AF.

La mayoría de las participantes $(99,7 \%)$ dijeron que estarían interesadas en recibir AF durante una nueva gestación y que $57,9 \%$ preferiría hacerlo a 


\section{Tabla 2. Principales métodos referidos por las encuestadas para prevenir la aparición de malformaciones congénitas}

\begin{tabular}{|lr|}
\hline Método* $^{*}$ & $\%$ \\
\hline Evitar drogas, alcohol y tabaco & 32,1 \\
Tomar ácido fólico & 25,9 \\
Tomar vitaminas & 8,6 \\
Dieta balanceada & 4,9 \\
Control prenatal & 3,7 \\
Otros & 24,8 \\
\hline
\end{tabular}

*Podía haber una o más respuestas por encuestada

\section{Tabla 3. Conocimientos y uso de suplementación con AF en 342 mujeres puérperas en Santiago de Chile}

\begin{tabular}{|lrr|}
\hline & $\mathrm{N}$ & $\%$ \\
\hline Ha ó́do sobre el AF: & & \\
$\quad$ SI & 162 & 47,4 \\
$\quad$ NO & 180 & 52,6 \\
Sabe que el AF previene & & \\
DTN: & 33 & 9,6 \\
$\quad$ SI & 309 & 90,4 \\
$\quad$ NO & & \\
Fuente de información & & \\
sobre el AF*: & 116 & 34,0 \\
$\quad$ Matronas & 97 & 28,0 \\
$\quad$ Medios de comunicación & 68 & 20,0 \\
$\quad$ Médicos & 33 & 9,6 \\
$\quad$ Amigos & 27 & 8,0 \\
Familiares & & \\
Tomó AF en su último & & \\
embarazo: & 57 & 17,0 \\
$\quad$ SI & 285 & 67,0 \\
$\quad$ NO & & \\
Comenzó a tomar AF: & 10 & 2,9 \\
$\quad$ Pregestacionalmente & & \\
Cuando supo que estaba & 47 & 13,7 \\
$\quad$ embarazada & & \\
\hline
\end{tabular}

AF: Acido fólico, *Puede ser una o más respuestas por encuestada, DTN: Defecto de cierre de tubo neural. través de la fortificación de los alimentos con dicha vitamina.

Respecto al consumo de pan $78 \%$ de las mujeres encuestadas ingería una cantidad suficiente para asegurar la ingesta de la dosis de AF recomendada internacionalmente para prevenir la ocurrencia de DTN en personas sin factores de riesgo.

Respecto a controles realizados en la atención primaria, previos a su embarazo, setenta y tres mujeres $(21,3 \%)$ efectuaron consultas, en la mayoría de los casos para informarse sobre métodos anticonceptivos; y doscientas cincuenta y cuatro encuestadas $(74,3 \%)$ manifiestaron estar interesada en realizar una cita de control pregestacional antes de un nuevo embarazo.

Los estudios de correlación univariado y multivariado de Pearson mostraron como datos estadísticamente significativos que: las mujeres entre 25-34 años tenían mayor conocimiento acerca del AF y su rol en la prevención de anomalías congénitas y que fueron quienes más utilizaron correctamente el AF (valor de p: 0,02); por otra parte se encontró que las mujeres que habían realizado algún control preconcepcional en la atención primaria tenían mayor conocimiento sobre el AF (valor de p: 0,03). Si bien no fueron significativas las diferencias, se encontró que proporcionalmente era mayor el conocimiento del AF por parte de las mujeres con mayor nivel de educación y mayor número de gestaciones.

\section{DisCUSIÓN}

Los hallazgos de este estudio muestran que $47,4 \%$ de las mujeres puérperas encuestadas han oído hablar sobre el AF. De acuerdo a los datos comunicados en la literatura, este nivel basal de conocimiento es mayor comparado con China (36\%); similar a Canadá (50\%) y menor que Israel (84\%), Estados Unidos de Norteamérica (79\%), Croacia (72\%) y Holanda $(60-85 \%)^{6,8,12-15}$.

Además, sólo 9,6\% de la muestra reconoce el papel del AF en la prevención de defectos congénitos, lo cual es menor frente a países como Irlanda, China, Estados Unidos de Norteamérica y Noruega; en los cuales el nivel de conocimiento de esta asociación oscila entre 21 y $77 \% 12,16-18$. 
En países como Holanda (11,5 DTN por cada 10000 fetos $^{19}$, Israel (11-22,3 DTN por cada 10000 recién nacidos vivos) ${ }^{20}$, Noruega (11,9 DTB por cada 10000 recién nacidos vivos) ${ }^{10}$, Estados Unidos (30,5 DTN por cada 100000 recién nacidos vivos) ${ }^{21}$ e Irlanda (24 DTN por cada 10000 fetos) ${ }^{19}$, se han realizado campañas educativas sobre el AF, logrando con dicha intervención que la cantidad de mujeres que reconocen el AF y sus cualidades se incremente desde $7 \%$ hasta $29 \% 6,8,15,18$. Una causa del bajo nivel de conocimiento del AF en las mujeres encuestadas en este trabajo, podría ser que en Chile no haya campañas masivas de promoción de la ingesta de $\mathrm{AF}$, como si existen en otras latitudes.

Los datos indican que la mayoría de las mujeres (83\%) no tuvieron la recomendación especial de suplementación con ácido fólico para prevenir DTN y que sólo 2,6\% la recibieron de manera adecuada. Estos resultados son similares a la situación estadounidense previa a las campañas educacionales, donde la encuesta de aplicación de la suplementación periconcepcional de AF por parte del personal de la atención primaria reportó $10 \%$ de adherencia ${ }^{1}$, porcentaje que tras las campañas educativas se incrementó a $29 \% 8$.

La importancia de lograr una mayor difusión de esta información en mujeres en edad reproductiva, radica en el hecho informado ya por otros autores de que las mujeres que conocen sobre las ventajas del uso periconcepcional del AF presen$\tan$ incrementos en su utilización entre 6 y 50 veces más que aquellas que no poseen conocimientos sobre el tema ${ }^{6-7}$. Por otra parte, en este estudio se encuentra que la ingesta de alimentos fortificados (pan) estaría resultando en un incremento de al menos 360 $\mathrm{ug}$ de AF diario en la dieta del $78 \%$ de las mujeres encuestadas, lo cual es similar a otros estudios recientes en Chile ${ }^{22-23}$.

Chile ha logrado reducir a 1:1.000 el nacimiento de niños nacidos afectados con DTN tras el inicio del programa de fortificación de la harina de trigo con $\mathrm{AF}$, es decir $40 \%$ de reducción frente al período prefortificación ${ }^{2}$. Para disminuir aún más este porcentaje, existe desde 2003 una norma de suplementación con AF dirigida a aquellas mujeres que no alcanzan a suplir sus requerimientos de AF con el programa de fortificación, pero los presentes resultados muestran que pocas mujeres reciben dicha suplementación adecuada- mente y lo que resulta más preocupante es que no recibieron suplementación las mujeres con alto riesgo para tener hijos afectados por DTN, correspondientes al $0,9 \%$ de nuestra muestra. Este dato concuerda con otro estudio conducido por nuestro grupo sobre la aplicación de la norma ministerial de suplementacion a nivel de la atención primaria, en el cual se demostró que sólo 5,1\% de los profesionales encuestados tenía conocimiento adecuado de dosis y período de uso del AF para la prevención de anomalías congénitas ${ }^{5}$. Esta norma nacional tenía solamente un año de vigencia al momento de la aplicación de la encuesta, lo cual, aunado a la falta de su promoción, podría explicar el pobre nivel de su aplicación.

Existen experiencias en la literatura que evidencian que campañas educativas comunitarias sobre el AF tienen poco impacto en el aumento de conocimientos sobre el mismo ${ }^{10,24}$, pero trabajos dedicados a capacitar simultáneamente a la comunidad y profesionales de la salud sobre este tema han logrado incrementar dicho conocimiento de manera significativa, entre 30 y $60 \%{ }^{24-26}$.

En este trabajo, las mujeres entre 25-34 años y aquellas que habían realizado algún control pregestacional, tenían mayor conocimiento sobre AF, lo cual es concordante con lo comunicado en China e Israel ${ }^{6,12}$. En nuestra población, al menos $21,3 \%$ de las mujeres tuvieron una consulta preconcepcional, por tanto la atención primaria sería una buena instancia para enseñar sobre las medidas de prevención primaria de anomalías congénitas, comprendidas en el decálogo del Estudio Colaborativo Latinoamericano de Malformaciones Congénitas (ECLAMC) ${ }^{27}$.

Si bien nuestros datos no son significativos en este aspecto, muestran la tendencia a que un mayor nivel de educación se relaciona con un mejor conocimiento sobre AF, lo cual ha sido descrito por otros autores en Israel, Croacia y Holanda $6,14,15$. En este trabajo las diferencias podrían no ser aún significativas debido al bajo número de participantes con educación universitaria $(17,8 \%) y$, por ende, corresponder a un sesgo de muestra.

No obstante, ante la evidencia de un nivel medio de educación en las encuestadas, la mayoría de ellas han cursado parte o la totalidad de su educación secundaria o media, y considerando que $16 \%$ de los embarazos en Chile ocurren en 
adolescentes, educar en los colegios sobre este tema sería otra buena vía de formación.

Este estudio tiene sesgos, como la realización de las encuestas por personal calificado en el área (estudiantes de medicina), lo cual podría inducir de manera involuntaria un sesgo en las respuestas. Para tratar de reducir este efecto se generó el cuestionario con diferentes tipos de preguntas y preguntas control. Asimismo, el hecho de haber sido realizado en una ciudad específica de Chile y sólo en 4 hospitales públicos, confiere un sesgo de selección. Sin embargo, si se considera que en Santiago, capital de Chile, vive aproximadamente el $50 \%$ de la población del país, de acuerdo al último censo nacional y que en Chile $70 \%$ de la población es cubierta por el sistema público de salud y $30 \%$ por el sistema privado; los resultados de este trabajo representan un porcentaje importante de la población del país.

En conclusión, dados los escasos conocimientos sobre AF detectados en la población estudiada y las experiencias internacionales sobre el tema, consideramos que realizar campañas públicas de educa-

\section{REFERENCIAS}

1. Centers for Disease Control and Prevention. Folic acid and prevention of spina bifida and anencephaly- 10 years after the US public health service recommendation. MMWR 51 (RR13); 1-3.

2. HertrampF E, Cortés F. Folic acid fortification of wheat flour: Chile. Nutr Rev 2004; 62(6Pt 2): S44-8

3. Castilla e, Orioli i, lópez-Camelo J, Graca Dutra M, NAZER J. Preliminary data on changes in Neural Tubes prevalence rates after folic acid fortification in South America. Am J Med Genets 2003; 123A: $123-8$

4. Ministerio de Salud de Chile. XXX capítulo. $1^{\underline{a}}$ ed. Santiago. Guía Perinatal. Santiago, 2003: 359-86.

5. Pardo R, Lay-Son G, Aranda W, Recabal P, Navarrete M, Tenhamm T et al. Chilean primary health workers' knowledge about folic acid supplementation for the prevention of neural tube defects. Am J Med Genet A, 2006; 140: 1238-40.

6. Amitai Y, Fisher N, Haringman M, Meiraz H, Baram $\mathrm{N}$, LeVenthal A. Increased awareness, knowledge ción a la población general y los profesionales de la salud acerca del AF y la prevención de DTN podrían contribuir a reducir aún más la incidencia de los DTN lograda tras el programa de fortificación de la harina de trigo con AF en Chile. Sobre todo si se consideran como grupos poblacionales no cubiertos con dicho programa y candidatos a recibir suplementación a: aquellas mujeres con baja ingesta de harinas (no reciben la dosis óptima de AF a través del programa de fortificación) y las que poseen con factores de riesgo para DTN (por requerir más dosis de la que se ofrece a través del programa de fortificación en Chile).

\section{Agradecimientos}

Los autores agradecen a todas las personas que colaboraron en la investigación: a autoridades administrativas y el personal clínico de los Departamentos de Ginecología y Obstetricia del Hospital Clínico Universidad de Chile, Hospital San Juan de Dios, Hospital Barros Luco, Hospital Luis Tisné y Hospital San Borja Arriarán. and utilization of periconceptional folic acid in Israel following a national campaign. Prev Med 2004; 39: 731-7.

7. Coll O, Pisa S, Palacio M, Quinto L, Cararach V. Awareness of the use of folic acid to prevent neural tube defects in Mediterranean area. Eur J Obstet Gynecol Reprod Biol 2004; 115: 173-7.

8. MOD, 2003. March of Dimes. Folic acid and the prevention of birth defects: a national survey of pre-pregnancy awareness and behavoir among women of childbearing age, 1995-2003. The Gallup Organization.

9. Bonin MM, Bretzlaff JA, Therrien SA, Rowe BH. Knoledge of periconceptional folic acid for the prevention of neural tube defects. The missingn links. Northeastern Ontario Primary Care Research Group. Arch Fam Med 1998; 7: 438-42.

10. Braekke K, Staff A. Periconceptional use of folic acid supplements in Oslo. Acta Obstet Gynecol Scand 2003; 82: 620-7.

11. Abdulrazzaq YM, Al-Gazali li, Beber A, Hossein M, Verghese M, Dawodu A et al. Folic acid awareness 
and intake survey in the United Arab Emirates. Reprod Toxicol 2003; 17: 171-6.

12. Ren A, Khang L, Li Z, HaO L, Tian Y, Li Z. Awareness and use of folic acid, and blood folate concentrations among pregnant women in northern China - An area with a high prevalence of neural tube defects. Reprod Tox 2006; 22: 431-6.

13. French M, Barr S, LeVY-Milne Ryna. Folates intakes and awareness of folate to prevent neural tube defects: A survey of women living in Vancouver, Canada. J American Dietetic Association 2003; 130: 181-5.

14. Gjergja R, Stipoljev F, Hafner T, Tezak N, LuzarSTIFFLER V. Knowledge and use of folic acid in Croatian preganant women - a need for health care education initiative. Rep Tox 2006; 21: 16-20.

15. Walle HeK, Cornell MC, De Jong-Van den Berg LTW. Three years after Dutch folic acid campaign: Growing socioeconomic differences. Prev med 2002; 35; 65-9.

16. Ward M, Hutton J, Mc Donell R, Bachir N, Scallan E, Oleary M et al. Folic Acid supplements to prevent neural tube defects: Trends in East of Ireland 1996-2002. Ir Med J 2004; 97: 274-6.

17. Canfiel Ma, Przybyla SM, Case aP, Ramadhani T, SuAREZ L, Dyer J. Folic acid awareness and suplementation among Texas women of childbearing age. Prev Med 2006; 43: 27-30.
18. KJersti A, Emil S, lande B, Oien H. 2004. Changes in knowledge and attitudes of folate, and use of dietary supplements among women of reproductive age in Norway 1998-2000. Scand J Public Health; 32: 264-71.

19. Olivares S, Vío F. Estudio comparativo de la dieta de los chilenos con la dieta mediterránea. Rev Chil Nutr 2001; 28: 269-76.

20. Vío F, Abdala C. Nutrition policy in the Chilean transition. Public Health Nutr 2000; 3: 49-55.

21. Centers for Disease Control and Prevention. Knowledge and use of folic acid by women of childbearingage- United states, 1995 and 1998. MMWR 1999; 48: 325-7.

22. De Jong-Van den Berg LT, Hernández-Díaz S, Werler MM, Louis C, Mitchell AA. 2005. Trends and predictors of folic acid awareness and periconceptional use in pregnant women. $A m J$ Obstet Gynecol 192, 12- 128.

23. Miranda A, Dávilia R, Gorrin J, Montes De Longo i. Puerto Rican primary physician's knowledge about folic acid supplementation for the prevention of neural tube defects. Birth Defects Research (part A): Clinical molecular teratology 2003; 67: 971-3.

24. Nazer, J. Prevención Primaria de Defectos Congénitos. Rev Méd Chil 2004; 132: 502-6. 